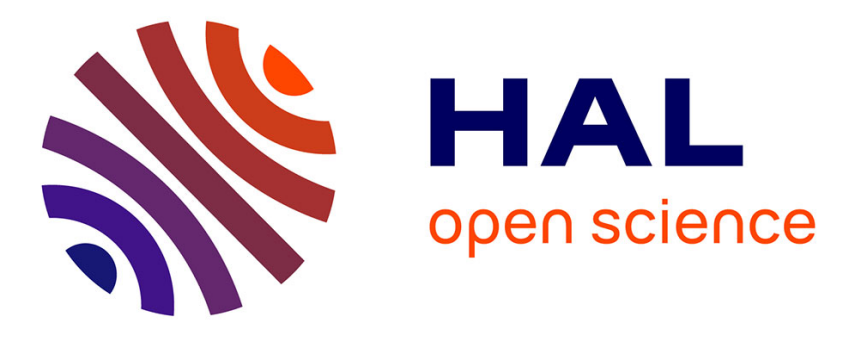

\title{
Quel bilan pour une gonarthrose avant prothèse Gérard Chales
}

\section{To cite this version:}

Gérard Chales. Quel bilan pour une gonarthrose avant prothèse. Revue du Rhumatisme monographies, 2016, Pathologies du genou, 83 (3), pp.166-171. 10.1016/j.monrhu.2016.04.003 . hal-01308002

\section{HAL Id: hal-01308002 https://hal-univ-rennes1.archives-ouvertes.fr/hal-01308002}

Submitted on 22 Jul 2016

HAL is a multi-disciplinary open access archive for the deposit and dissemination of scientific research documents, whether they are published or not. The documents may come from teaching and research institutions in France or abroad, or from public or private research centers.
L'archive ouverte pluridisciplinaire HAL, est destinée au dépôt et à la diffusion de documents scientifiques de niveau recherche, publiés ou non, émanant des établissements d'enseignement et de recherche français ou étrangers, des laboratoires publics ou privés. 


\section{Quel bilan pour une gonarthrose avant prothèse}

Which work up before a knee arthroplasty for knee osteoarthritis ?

Article in press: Revue du Rhumatisme Monographies (Rev Rhum Monogr) 2016, http://dx.doi.org/10.1016/j.monrhu.2016.04.003

Gérard Chalès $^{\mathrm{ab}}$,

a) Service de rhumatologie, hôpital sud, CHU de Rennes, 16, boulevard de Bulgarie, BP 90347, 35203 Rennes cedex 2, France

b) Université de Rennes 1, 2 Avenue du Professeur Léon Bernard, 35043 Rennes, France

Auteur principal et correspondant

Gérard Chalès, service de rhumatologie, Hôpital sud, CHU de Rennes

E-mail : gerard.chales@chu-rennes.fr

3800 mots

Mots clés : genou ; prothèse totale de genou ; bilan préopératoire ; examen clinique ; facteurs de risque; comorbidités ; anesthésie ; chirurgie orthopédique ; décision médicale partagée

Key Words: knee; total knee arthroplasty; preoperative work-up; physical examination ; risk factors ; comorbidities : anesthesia ; orthopaedic surgery ; shared decision-making

«Déclaration d'intérêts : Les auteurs ne déclarent aucun conflit d'intérêt. » 
Résumé

Le bilan pour une gonarthrose avant prothèse fait partie d'un parcours de soins qui devrait être centré sur le patient, nécessitant une meilleure concertation interdisciplinaire pour informer le patient de la balance bénéfices/risques liés à la pose d'une prothèse de genou et obtenir un accord mutuel entre le patient et les professionnels de santé, caractéristique essentielle du processus de "décision partagée » ou de "révélation des préférences des patients ». Cette décision repose sur les données de l'interrogatoire, la recherche des facteurs de risque généraux et des comorbidités, l'examen physique, l'évaluation de la gène fonctionnelle, le bilan radiologique, la recherche de foyers infectieux, l'évaluation des risques liés à l'anesthésie et à la chirurgie orthopédique, et l'information au patient

\section{Summary}

Work up before a total knee arthroplasty for knee osteoarthritis is part of a clinical care pathway which should be patient-centered, needing a best interdisciplinary communication and collaboration to inform patients about the balance benefits/risk linked to the knee arthroplasty and to obtain an agreement between patient and health's professionals, essential feature of the shared decision-making process or patient' preferences sensitive. This decision-making is based on the patient's history, search of general factors risk, comorbidities, physical examination, measure of knee function, radiographs, work-up for infection sources, assessment of risk linked to anesthesia and orthopaedic surgery, and delivering information to patients. 


\section{Introduction}

Les caractéristiques de la population française ayant eu une implantation d'une prothèse de genou (PTG) sont connues (63.5\% de femmes, âge moyen de 71 ans, $18 \%$ des patients avec une obésité morbide (IMC > 40)) selon une étude épidémiologique menée par l'Agence Nationale de Sécurité du Médicament (ANSM) à partir de la base de données médico-administratives de l'Assurance Maladie, et près de 90000 prothèses totales de genou ont été posées ou révisées en France en 2013 soit une augmentation de plus de 33\% par rapport à 2008 [1]. Cependant, il ne faut pas oublier que seulement $85 \%$ des patients ayant une PTG sont satisfaits des résultats de leur prothèse et que moins d'un patient sur deux a "oublié » qu'il a eu une intervention [2], contrairement à la mise en place d'une prothèse de hanche qui donne la plupart du temps une hanche " oubliée ». C'est dire l'importance du bilan préopératoire qui devra décrypter au mieux les "attentes" des patients et les traduire en objectifs « réalisables ».

Le bilan préopératoire avant prothèse du genou (PTG) est actuellement très (trop) segmenté entre les différents professionnels de santé, qu'il s'agisse du médecin généraliste [3], du rhumatologue [4], du chirurgien orthopédiste [5-6], de l'anesthésiste [7-8], et du médecin de médecine physique [9-10], face aux préférences individuelles et aux besoins des patients [11].

Ce bilan préopératoire fait partie d'un parcours de soins qui devrait être centré sur le patient [12], nécessitant une meilleure concertation interdisciplinaire pour informer le patient de la balance bénéfices/risques liés à la pose d'une PTG et obtenir un accord mutuel entre le patient et les professionnels de santé, caractéristique essentielle du processus de "décision partagée " ou de "révélation des préférences des patients " [13]. Cette décision repose sur les données de l'interrogatoire, la recherche des facteurs de risque généraux et des comorbidités, l'examen physique, l'évaluation de la gène fonctionnelle, le bilan radiologique, la recherche de foyers infectieux, l'évaluation des risques liés à l'anesthésie et à la chirurgie orthopédique, et l'information au patient (tableau 1).

\section{Interrogatoire}

L'interrogatoire se doit d'analyser en premier lieu la plainte fonctionnelle selon les caractéristiques de la douleur (début, facteur déclenchant, évolution dans le temps, efficacité des traitements) et l'autonomie permise. L'intensité de la douleur, mesurable par l'échelle visuelle analogique, n'est pas un critère de sélection pour l'indication chirurgicale, ou tout du moins, il n'a pas été démontré un seuil de douleur au-delà duquel une PTG serait justifiée selon les travaux du comité ORSI-OMERACT [14]. En revanche, l'intensité de la douleur préopératoire était prédictive d'une persistance de la douleur après PTG, tout comme la présence de catastrophisme, de détresse psychologique et de comorbidités selon une méta-analyse récente [15]. Le 
catastrophisme et la dépression ont une influence sur la douleur et la fonction, sans oublier que la douleur contribue à l'anxiété et à la dépression qui peuvent parfois s'améliorer grandement après la chirurgie prothétique. L'état psychologique était un facteur prédictif de la satisfaction, de la douleur ou de la fonction 6 mois après la pose de la PTG selon une méta-analyse [16]. La "non réponse " aux traitements médicaux peut être un des facteurs de décision d'une PTG pour le chirurgien [17].

L'autonomie permise est bien mesurée par l'indice algofonctionnel de Lequesne: périmètre de marche, utilisation de cannes, difficultés à la pratique des escaliers et au passage de la station assise à la position debout. La capacité en préopératoire de monter et de descendre l'escalier sans l'aide de la rampe était le meilleur facteur prédictif (suivi par le jeune âge et une plus grande force du quadriceps) pour déterminer les individus qui n'auraient pas besoin de rampe après PTG [18].

Les antécédents peuvent avoir une influence sur la décision opératoire: gonarthrose post-traumatique, genou multi-opéré, arthrodèse du genou ou de la hanche, arthrite septique active, déficit du quadriceps (maladie neurologique), algodystrophie récente [6].

Les attentes des patients doivent être clairement définies [19] et certains attendent beaucoup de l'intervention [20], de façon irréaliste, ce d'autant qu'ils sont plus jeunes, plus physiquement actifs, plus demandeurs d'une récupération rapide et d'un retour à l'état fonctionnel antérieur leur permettant la reprise des activités professionnelles, de loisir et/ou sportives. II faut savoir modérer cet optimisme et expliquer que le soulagement de la douleur, la capacité de marcher et d'effectuer les activités de la vie quotidienne sont des attentes réalistes. Ces attentes sont prises en considération, tout comme le niveau de satisfaction et la qualité de vie lors de la pratique des activités de loisir et/ou sportives, dans des questionnaires autoadministrés (recueillant les données rapportées par les patients) issus de l'adaptation française du nouveau score de la Knee Society pour la PTG [21] ou du Knee Replacement Expectations Survey [22].

Les conditions de vie (habitat, seul, couple) doivent être précisées pour " prévoir » le retour à domicile dans de bonnes conditions après l'intervention.

\section{Recherche des facteurs de risque généraux}

\subsection{Age}

L'âge n'est pas un facteur limitant pour la pose d'une PTG et même après 80 ans on observait certes une amélioration des douleurs et du Knee Society Score mais une moins bonne fonction et plus de complications [23].

\subsection{Etat cardiovasculaire}


L'état cardiovasculaire doit être évalué (anesthésiste, cardiologue). La chirurgie prothétique est associée à une morbidité postopératoire, incluant les accidents cardiaques ischémiques périopératoires, en particulier dans une population vieillissante avec des comorbidités préexistantes à l'intervention [24]. Les anesthésistes connaissent bien, en chirurgie orthopédique, le risque de décès et d'infarctus du myocarde non fatal augmenté en postopératoire immédiat [25], en cas d'antécédents cardiorespiratoires, d'hypertension artérielle, d'antécédents d'accident vasculaire cérébral et de diabète. Le risque de complication cardiaque (mort d'origine cardiaque, infarctus du myocarde) durant la période périopératoire peut être stratifié en considérant des facteurs liés au patient et le type de chirurgie. Cet algorithme clinique a été développé par l'AHA (American Heart Association) et l'ACC (American College of Cardiology) [26].

\subsection{Etat vasculaire périphérique}

L'état vasculaire périphérique doit aussi être précisé, conduisant à rechercher la notion de claudication intermittente, de douleurs au repos, d'antécédents de chirurgie vasculaire ; à chercher à l'examen clinique la présence des pouls (creux poplité) et en cas de doute à faire un indice de pression systolique (cheville, artère humérale) qui pourra conduire à des investigations complémentaires [27]. Concernant l'état veineux [28-29], on connaît mieux actuellement les facteurs de risque, chez le sujet âgé, de maladie veineuse thromboembolique, à savoir l'insuffisance cardiaque congestive, la bronchopathie chronique obstructive et le diabète.

\subsection{Diabète}

Le diabète a une influence sur les complications de la PTG. Les patients diabétiques avaient une augmentation du risque d'infection profonde [OR: 1,$6 ;$ IC 1,4-1,9], de thrombose veineuse $[2,6 ; 1,6-4,2]$, de fracture périprothétique $[1,9 ; 1,0-3,4]$, de descellement aseptique $[9,4 ; 4,6-18,9]$ et d'un moins bon score fonctionnel dans une méta-analyse récente portant sur 835071 PTG [30]. Les complications seraient plus fréquentes chez les diabétiques insulino-requérant [31].

\subsection{Obésité}

L'obésité est source d'une augmentation des complications postopératoires selon l'association américaine des chirurgiens de la hanche et du genou [32] ; ce résultat doit être tempéré par le registre anglais des prothèses (British National Joint Registry) [33] dans lequel on utilise les critères rapportés par le patient et des questionnaires auto-administrés qui reproduisent plus fidèlement le ressenti des patients ; avec le questionnaire d'Oxford adapté au genou, on a une amélioration identique chez les obèses et les non obèses, indépendante de I'IMC, avec cependant une augmentation du taux de complications (cicatrisation) : 17\% (IMC 40$60)$ vs $9 \%(\mathrm{IMC}<25)[33]$. 
Ainsi, la relation entre l'IMC et le devenir de la PTG reste débattue. Cependant, l'obésité avait une influence négative sur les résultats de la PTG dans une 1 ère métaanalyse de 2012 [34] où les 2 complications suivantes étaient plus fréquemment rencontrées chez les obèses : l'infection (OR: 2,4 ; IC 1,3-4,5), la révision de la PTG $(1,3 ; 1,0-1,7)$. Ces données ont été confirmées dans une $2^{\text {ème }}$ méta-analyse de 2015 [35], montrant que la révision (avec un suivi > 5ans), l'infection du site opératoire, et la thrombose veineuse profonde survenaient plus fréquemment chez les patients obèses, l'infection profonde plus fréquemment dans l'obésité morbide. II y a effectivement un consensus pour dire que pour un IMC >40, il existe une augmentation très significative des complications postopératoires, et en particulier de l'infection lorsqu'on conjugue diabète et obésité ; en effet le taux annuel d'infections prothétiques était de $0,72 \%$ dans un centre finlandais (7181 PTH/PTG) de 2002 à 2008 , corrélé à l'IMC $(0,37 \%$ pour un IMC normal vs $4,66 \%$ pour un IMC > 40$)$, en soulignant que le diabète multiplie par 2 le risque d'infection, indépendamment de l'obésité [36]. II faut en avertir les patients.

Doit-on encourager une perte de poids dans l'année qui précède la pose de la PTG (avec à la fois des conseils diététiques et une augmentation de l'activité physique pas toujours possible) ? En théorie oui, sachant qu'il existe trop peu de preuves convaincantes en faveur de l'efficacité de la perte de poids, y compris par chirurgie bariatrique [37], et que l'infection du site opératoire pourrait être favorisée chez les obèses ayant perdu plus de $5 \%$ de leur poids (rôle de la malnutrition ?) [38]. II faut savoir aussi que les patients qui venaient d'avoir une PTG ne perdaient ou ne gagnaient pas plus de poids que les patients gonarthrosiques non opérés [39], ce qui veut dire que la PTG ne résout pas à elle seule le problème de l'obésité. Les patients doivent en être avertis.

\subsection{Tabac}

Le tabac a une action délétère sur le système musculo-squelettique [40]; il peut interférer avec le phénomène de cicatrisation et augmenter le risque d'infection pour de multiples raisons (vasoconstriction, réaction fibroblastique, hypoxie tissulaire) [41]. Les fumeurs actuels avaient une augmentation du risque d'infection profonde de I'incision (OR: 1,$5 ;$ IC 1,2-1,8) et des complications $(1,2 ; 1,0-1,3)$, tout comme les anciens fumeurs $(1,2 ; 1,0-1,3)$ dans une analyse multivariée concernant 78191 patients, issus d'une base de données du collège américain des chirurgiens [42], ayant eu une PTH ou une PTG, 10,3\% étant des fumeurs actuels et $7,9 \%$ des anciens fumeurs ; l'augmentation du nombre de paquets-année $(>40)$ entraînait une augmentation du risque de complications totales $(1,2 ; 1,0-1,4)$ [42], suggérant un effet systémique du tabac. Les fumeurs actuels de la Mayo Clinic (565/7926 patients porteurs de PTG ou PTH) avaient aussi une augmentation du risque d'infection profonde $(2,4 ; 1,2-4,7)$ et de révision de l'implant $(1,8 ; 1,0-3,1)$ [41].

Lorsqu'on comparait les genoux des fumeurs aux genoux des non fumeurs, on avait essentiellement des révisions qui étaient non septiques (13/131 vs $5 / 590$, suivi de 46 mois), avec une augmentation du taux de complications postopératoires chez les 
fumeurs, qu'elles soient chirurgicales ( $5 \%$ vs $2 \%$ ) ou médicales ( $21 \%$ vs $12 \%$ ) [43]. Les facteurs liés au patient, indépendamment associés à la révision, étaient le tabac (OR: 2,$9 ; 1,3-6,2)$ et le sexe masculin $(1,7 ; 1,0-2,8)$ dans une étude -cas-témoins [44].

Le tabac était prédictif des complications post-opératoires lorsqu'on comparait, dans une série de 33336 patients (95\% d'hommes) ayant eu une PTG ou PTH, des patients qui n'avaient jamais fumé $(57 \%)$ à des patients qui avaient arrêté de fumer (19\%) ou qui fumaient encore (24\%): pour ces derniers, le risque relatif était augmenté pour le risque d'infection du site opératoire $(1,4 ; 1,2-1,7)$, d'accident vasculaire cérébral $(2,6 ; 1,3-5,4)$, de pneumonie $(1,5 ; 1,1-2,1)$ et de décès $(1,6$; $1,3-2,0)$ à 1 an [45].

L'arrêt du tabac avant la chirurgie diminuait le taux de complications, avec une réduction importante des actes chirurgicaux secondaires (- 77\%), des complications de cicatrisation (- 84\%) ou de toutes complications (- 66\%) [46]. C'est dire l'importance de toute intervention susceptible d'aider le patient à s'arrêter de fumer au moins 6 semaines avant et après l'intervention.

\section{Recherche de comorbidités}

Les comorbidités médicales, en dehors des facteurs de risque généraux, chez les sujets candidats à une prothèse (PTH, PTG) ont été étudiées à partir de la base de données Osteoarthitis Initiative [47] : les plus fréquentes étaient l'ostéoporose (16\%), la dépression légère ou modérée $(8 \%)$, le cancer $(8 \%)$, le diabète $(6 \%)$, un antécédent d'accident vasculaire cérébral $(6 \%)$ et l'asthme $(5 \%)$. Si ces maladies sont fréquemment rencontrées à l'âge moyen de pose d'une prothèse (70 ans), elles peuvent avoir un effet délétère sur les résultats postopératoires, augmenter potentiellement le risque de complications et réduire la capacité d'activité physique, à court et à long terme après l'intervention; le programme de rééducation devra en tenir compte, ainsi que les conseillers médicaux qui devront prendre en charge ces comorbidités pour lesquelles il faut encourager et maintenir les programmes d'activité physique. II existe des outils fiables et validés permettant d'évaluer ces comorbidités: index de comorbidité fonctionnelle, index de Charlson (Annexe A tableau S1 et S2) [48].

\section{Examen physique}

Un examen clinique est nécessaire afin d'évaluer [6], (voir Legre \& Boyer [49], dans ce numéro):

- la démarche, le passage du pas, l'axe des membres inférieurs ; 
- la localisation précise de la douleur ressentie par le patient : tester chaque compartiment (fémorotibial médial et latéral, fémoropatellaire, tibiofibulaire) ;

- la mobilité du genou en flexion (lors de l'accroupissement, de la montée ou de la descente de la table d'examen), l'existence d'un flexum (perte de l'extension: différencier un flexum passif qui est un défaut d'extension fixé, d'un flexum actif, qui est un défaut d'extension par insuffisance du quadriceps), d'un recurvatum ;

- la stabilité du genou (pivot central, stabilité frontale, laxité d'usure, réductibilité d'un varus ou d'un valgus);

- l'état vasculaire (voir 3.2) ;

- l'état cutané et l'on connaît bien les facteurs de risque cutané actuellement : corticothérapie au long cours, diabète, artériopathie des membres inférieurs, tabagisme, mauvais état veineux, genou multi-opéré (multi-cicatriciel) ;

- la mobilité de la hanche et du rachis (douleur référée dans le cadre d'un canal lombaire rétréci).

\section{6. Évaluation de la gêne fonctionnelle}

On utilise habituellement l'échelle visuelle analogique (EVA) de la douleur en 100 $\mathrm{mm}$ et l'indice algofonctionnel de Lequesne. Le score du WOMAC est le premier score de qualité de vie spécifique rempli par le patient, suivi en 2003 par le Knee injury and Osteoarthritis Outcome Score (KOOS) [collins], incluant une analyse plus fine et plus complète de la qualité de vie du patient et de ses aptitudes sportives. Actuellement, de nombreux scores sont à la disposition du rhumatologue pour évaluer la fonction du genou [50]. Le plus utilisé et le plus consensuel à ce jour est sans doute le score de l'International Knee Society (IKS) de 1989, avec ses deux composantes clinique et fonctionnelle, revu en 2012, avec ajout d'un score "subjectif» se présentant sous la forme d'un questionnaire auto-administré évaluant la fonction du genou à travers les activités propres à chaque patient [21]. Les questionnaires auto-administrés permettent d'obtenir les " critères d'évaluation rapportés par le patient » ou patient-reported outcomes (PRO), méthode fiable aujourd'hui très employée dans l'évaluation des résultats chirurgicaux, notamment déjà utilisée dans le score d'Oxford adapté en français (Annexe A, tableau S3) [51].

\section{Le bilan radiologique}

Bien qu'il n'existe pas de consensus sur le bilan radiologique "standard " (fonction des questions posées par le prescripteur) avant PTG, celui-ci comportera:

- un cliché des 2 genoux de face debout (en extension ou/et en schuss), profil couché à $30^{\circ}$ de flexion, pour vérifier le stade de l'arthrose, l'atteinte uni ou tricompartimentale, la position d'une cupule, la pente tibiale ; 
- une incidence fémoro-patellaire (défilé fémoro-patellaire à $30^{\circ}$ de flexion pour juger du centrage rotulien, à $60^{\circ}$ pour apprécier une éventuelle arthrose fémoropatellaire ; - des clichés en stress (de face en varus et valgus forcé), pour vérifier la réductibilité de la déformation ;

- une télémétrie (pangonogramme) debout de face (en appui bi-podal, en appui unipodal sur le genou concerné) permettant la mesure de l'axe fémorotibial mécanique dans le plan frontal du genou opéré et controlatéral ;

- une radiographie du bassin de face debout: une arthrose associée de la hanche, symptomatique, ferait préférer la prise en charge initiale de celle-ci.

Le système de radiographie EOS, 7 à 10 fois moins irradiant qu'une radiographie classique, permet d'acquérir dans le même temps le cliché de face et de profil et de faire des reconstructions $3 \mathrm{D}$ utiles pour analyser les déformations les plus sévères des cas difficiles [52].

Tous ces clichés permettent de mesurer le grade d'usure, de préciser l'état des compartiments et des ligaments collatéraux. Une IRM n'est pas indiquée, sauf s'il existe un doute sur le ligament croisé antéroexterne.

Les patients qui avaient des lésions radiographiques plus sévères avant la chirurgie étaient plus susceptibles d'avoir une amélioration notable en termes de soulagement de la douleur et d'amélioration de la fonction par la pose de la PTG [53], ce qui veut dire que les chirurgiens devront être particulièrement prudents dans la décision d'opérer un patient avec des gonalgies intenses associées à des signes radiographiques modestes de gonarthrose, ce d'autant qu'il existe des problèmes psycho-sociaux.

\section{Recherche de foyer infectieux}

L'éradication des foyers infectieux est souhaitable chez tout candidat à une prothèse articulaire car sources de contamination hématogène. Le bilan préopératoire classique comporte la recherche de lésions cutanées au voisinage ou à distance, une consultation ORL et stomatologique, un dosage de la CRP, un ECBU (10 à 15 jours avant l'intervention).

Le bilan dentaire (par un dentiste et/ou un stomatologiste pour le parondonte) est généralement exigé en France avant la pose d'une prothèse, pour éliminer une infection dentaire active, bien qu'il y ait peu d'arguments pour justifier cette pratique dans la littérature: cependant $80 \%$ des participants à une conférence américaine de consensus en 2013 sur l'infection des prothèses ont voté en faveur d'un dépistage de l'infection dentaire active chez les candidats à une prothèse articulaire ; celui-ci serait notamment indispensable chez les patients âgés, intoxiqués (tabac, stupéfiants), avec une mauvaise hygiène bucco-dentaire ou des antécédents d'extraction dentaire. [54]. 
Bien qu'aucune donnée solide n'ait été produite pour les bactériuries asymptomatiques, un dépistage systématique à la recherche d'une bactériurie asymptomatique ainsi que son traitement sont largement pratiqués, notamment en France ; or dépister et traiter les BAS avant la pose d'une prothèse articulaire chez des patients sans facteur de risque ne semble pas utile pour prévenir une infection de prothèse [55].

II faut rappeler aux patients que de multiples facteurs potentiellement modifiables (tabac, obésité (IMC > 30), diabète, dépression, corticothérapie) sont associés à un risque d'infection de la prothèse à long terme [56]. Un score pour évaluer le risque endogène d'infection du site opératoire a été développé par une équipe française mais non encore validé [57].

\section{Consultation d'anesthésie}

L'anesthésiste va expliquer au patient les différents type d'anesthésie (générale, rachidienne) et l'intérêt éventuel d'une anesthésie locorégionale tronculaire à visée analgésique postopératoire, en fonction du type de chirurgie, de sa durée, du terrain du patient, du bilan préopératoire et du désir du patient, avec les avantages et les inconvénients inhérents à chaque technique. L'évaluation du risque lié à l'anesthésie sera discutée lors de cette consultation en utilisant la classification ASA (Annexe A, tableau S4) ; ce score a aussi une valeur pronostique après la pose d'une prothèse (PTH, PTG) [7]. La consultation permettra l'appréciation du terrain (extension du bilan usuel si besoin : état cardiorespiratoire, digestif, hépatique, rénal, urinaire), et la programmation éventuelle d'une autotransfusion. La prise en charge de la douleur post-opératoire sera abordée [8].

\section{Consultation chirurgicale}

Le chirurgien va exposer au patient les différentes options thérapeutiques, notamment chirurgicales, les raisons en faveur de la pose d'une prothèse de genou soit totale (PTG) soit unicompartimentale (PUC) (voir Belkaious et al. [58] dans ce numéro), le type d'implant utilisable, la voie d'abord (classique, miniinvasive), les difficultés opératoires liées au stade et au type d'arthrose, au terrain et à l'état local [6,59]. L'évaluation du risque lié à la chirurgie orthopédique sera discutée: saignement périopératoire (risques propres à la transfusion sanguine), événement thromboembolique (risques liés aux anticoagulants), risque infectieux (infection nosocomiale), dysfonctionnement cognitif. Les suites opératoires, la rééducation, les complications à court, moyen et long terme, le suivi clinique et paraclinique, les bénéfices espérés, la possibilité du retour aux activités sportives (voir Le Goux [60] dans ce numéro) devront être précisés.

\section{1.l'information du patient}


L'information préopératoire doit être comprise et loyale, et ce d'autant que plusieurs professionnels de santé interviennent [3], ce qui ne clarifie pas forcément les messages concernant la balance bénéfices/risques pour le patient. Si l'information orale est privilégiée, il faut pouvoir apporter la preuve de l'information donnée en cas de litige: d'où l'utilité de documents écrits explicatifs de l'intervention et des complications, voire d'un consentement écrit signé [61]. II faut laisser au patient un délai de réflexion afin qu'il puisse évaluer sereinement les risques et les bénéfices possibles de l'intervention chirurgicale proposée et prendre «sa » décision.

Le processus de décision d'un patient candidat éventuel à une PTG a été analysé dans une revue systématique [62] ; la relation de confiance avec le ou les praticiens est essentielle car le patient s'interroge sur l'incertitude des résultats d'une PTG (peur de l'intervention, des suites opératoires, de la douleur) et préfère parfois suivre les conseils (dissuasifs) de l'environnement social ou des médecines alternatives, expliquant certaines divergences d'opinion entre patient et praticiens [11]. Dans ce processus, il y a une phase de délibération (conflit décisionnel source de stress ? retour en arrière si la symptomatologie s'améliore) jusqu'à ce que la décision de se faire opérer soit prise. Des aides à la décision (vidéos interactives, livrets, témoignages de patients, vignettes) amélioraient les connaissances du patient, entraînaient moins de décision conflictuelle, moins d'indécision et une plus grande concordance entre les valeurs des patients et les options choisies [63]. Faut-il envisager une éducation et une rééducation préopératoire ? Oui, si elle peut contribuer à réduire la durée de séjour et à modifier les modalités de retour à domicile [10]. Les revues systématiques de 2012 [9], 2014 [64] et 2016 [65] n'ont pas apporté d'éléments convaincants suffisant pour mettre en œuvre en préopératoire soit une éducation, soit un programme de physiothérapie.

\section{Conclusions}

"Si l'arthroplastie est l'étape ultime dans la prise en charge d'un patient atteint d'une pathologie articulaire du genou, la décision d'intervention est dépendante de critères cliniques et radiologiques qui s'inscrivent dans un contexte lié au patient. L'intérêt de l'arthroplastie en regard des attentes du patient doit notamment être pris en compte. » [59]. C'est ce que l'on appelle une prise en charge personnalisée. Tous les éléments de ce bilan avant PTG ont pour but d'éclairer de façon précise la décision d'arthroplastie. C'est dire l'intérêt de développer un outil prédictif des résultats d'une PTG pour les patients candidats à un remplacement prothétique pour les aider à prendre leur décision [62], en dehors des aides à la décision et de leurs sources d'information. 


\section{Références}

1. ANSM. Surveillance des dispositifs médicaux à risque : Prothèses Totales de Genou (PTG). Janvier 2016.

2. Eymard F, Charles-Nelson A, Katsahian S, et al. "Forgotten knee" after total knee replacement: A pragmatic study from a single-centre cohort. Joint Bone Spine 2015;82:177-81.

3. Waugh EJ, Badley EM, Borkhoff CM, et al. Primary care physicians' perceptions about and confidence in deciding which patients to refer for total joint arthroplasty of the hip and knee. Osteoarthritis Cartilage 2015. doi: 10.1016/j.joca.2015.09.017.

4. Henrotin, Y, Chevalier X. Recommandations sur la prise en charge de l'arthrose de la hanche et du genou. Pour qui? Pourquoi? Pour quoi faire? Presse Méd 2010;39:1180-8.

5. Huten D. Chirurgie de la gonarthrose fémoro-tibiale. Rev Prat 2009;59:12-4.

6. Chatain F. La planification préopératoire dans les prothèses totales de genou. In: Bonnin M, Chambat C. La gonarthrose - Traitement chirurgical: de I'arthroscopie à la prothèse. Paris: Springer-Verlag;2006.p.247-64.

7. Rabuel C. Anesthésie et chirurgie orthopédique : y-a-t-il des limites ? In: Kahn MF, Bardin T, Dieudé P, Meyer O, Orcel P, Richette P. L'actualité rhumatologique 2013. Paris: Elsevier; 2013. p. 347-56.

8. Moucha CS, Weiser MC, Levin EJ. Current Strategies in Anesthesia and Analgesia for Total Knee Arthroplasty. J Am Acad Orthop Surg 2016;24:60-73.

9. Silkman Baker C, McKeon JM. Does preoperative rehabilitation improve patient-based outcomes in persons who have undergone total knee arthroplasty? A systematic review. PM R 2012;4:756-67.

10. Coudeyre E, Jardin C, Givron P, et al. Quel est l'intérêt d'une rééducation avant la pose d'une prothèse totale de hanche ou de genou? Élaborations de recommandations françaises pour la pratique clinique. Ann Réadapt Méd Phys 2007;50:179-88. 
11. Sansom A, Donovan J, Sanders C, et al. Routes to total joint replacement surgery: patients' and clinicians' perceptions of need. Arthritis Care Res 2010;62:1252-7.

12. Van Citters AD, Fahlman C, Goldmann DA, et al. Developing a pathway for high-value, patient-centered total joint arthroplasty. Clin Orthop Relat Res 2014;472:1619-35.

13. Haute Autorité de Santé. Patients et professionnels de santé : décider ensemble. Octobre 2013.

14.Gossec L, Paternotte S, Maillefert JF, et al.; OARSI-OMERACT Task Force "total articular replacement as outcome measure in OA". The role of pain and functional impairment in the decision to recommend total joint replacement in hip and knee osteoarthritis: an international cross-sectional study of 1909 patients. Report of the OARSI-OMERACT Task Force on total joint replacement. Osteoarthritis Cartilage 2011;19:147-54.

15. Lewis GN, Rice DA, McNair PJ, et al. Predictors of persistent pain after total knee arthroplasty: a systematic review and meta-analysis. $\mathrm{Br} \mathrm{J}$ Anaesth 2015;114:551-61.

16. Khatib Y, Madan A, Naylor JM, et al. Do Psychological Factors Predict Poor Outcome in Patients Undergoing TKA? A Systematic Review. Clin Orthop Relat Res 2015;473:2630-8.

17. Verra WC, Witteveen $K Q$, Maier $A B$, et al. The reason why orthopaedic surgeons perform total knee replacement: results of a randomised study using case vignettes. Knee Surg Sports Traumatol Arthrosc. 2016, doi 10.1007/s00167-015-3961-5.

18.Zeni JA Jr, Snyder-Mackler L. Preoperative predictors of persistent impairments during stair ascent and descent after total knee arthroplasty. J Bone Joint Surg Am 2010;92:1130-6.

19. Nizard R. Evolution de la prise en charge des patients nécessitant une prothèse totale de genou. Presse Méd 2010;39:1188-93. 
20.Smith EJ, Soon VL, Boyd A, et al. What Do Scottish Patients Expect of Their Total Knee Arthroplasty? J Arthroplasty 2015. doi: 10.1016/j.arth.2015.10.021.

21. Debette C, Parratte S, Maucort-Boulch D, et al. Adaptation française du nouveau score de la Knee Society dans l'arthroplastie du genou. Rev Chir Orthop Traumatol 2014;100:387-91.

22. Neuprez A, Delcour JP, Fatemi F, et al. Development and validation of the French version of a tool assessing patient's expectations in lower limb osteoarthritis. J Orthop 2014;12:46-57.

23. Kuperman EF, Schweizer M, Joy P, et al. The effects of advanced age on primary total knee arthroplasty: a meta-analysis and systematic review. BMC Geriatr 2016;16:41.

24. Ackland GL, Harris S, Ziabari Y, et al. Revised cardiac risk index and postoperative morbidity after elective orthopaedic surgery: a prospective cohort study. Br J Anaesth 2010;105:744-52.

25. Lu N, Misra D, Neogi T, et al. Total joint arthroplasty and the risk of myocardial infarction: a general population, propensity score-matched cohort study. Arthritis Rheumatol 2015;67:2771-9.

26. Fleisher LA, Fleischmann KE, Auerbach AD, et al. 2014 ACC/AHA guideline on perioperative cardiovascular evaluation and management of patients undergoing noncardiac surgery: executive summary: a report of the American College of Cardiology/American Heart Association Task Force on Practice Guidelines. Circulation 2014;130:2215-45.

27. Abu Dakka M, Badri H, Al-Khaffaf $\mathrm{H}$. Total knee arthroplasty in patients with peripheral vascular disease. Surgeon 2009;7:362-5.

28. Kapoor A, Labonte AJ, Winter MR, et al. Risk of venous thromboembolism after total hip and knee replacement in older adults with comorbidity and cooccurring comorbidities in the Nationwide Inpatient Sample (2003-2006). BMC Geriatr 2010;10:63.

29. Zhang J, Chen Z, Zheng J, et al. Risk factors for venous thromboembolism after total hip and total knee arthroplasty: a meta-analysis. Arch Orthop Trauma Surg 2015;135:759-72. 
30. Yang Z, Liu H, Xie X, et al. The influence of diabetes mellitus on the postoperative outcome of elective primary total knee replacement: a systematic review and meta-analysis. Bone Joint J 2014;96-B:1637-43.

31. Lovecchio F, Beal M, Kwasny M, et al. Do patients with insulin-dependent and noninsulin-dependent diabetes have different risks for complications after arthroplasty? Clin Orthop Relat Res 2014;472:3570-5.

32. Workgroup of the American Association of Hip and Knee Surgeons Evidence Based Committee. Obesity and total joint arthroplasty: a literature based review. J Arthroplasty 2013;28:714-21.

33. Baker $\mathrm{P}$, Petheram $\mathrm{T}$, Jameson $\mathrm{S}$, et al. The association between body mass index and the outcomes of total knee arthroplasty. J Bone Joint Surg Am 2012;94:1501-8.

34. Kerkhoffs GM, Servien E, Dunn W, et al. The influence of obesity on the complication rate and outcome of total knee arthroplasty: a meta-analysis and systematic literature review. J Bone Joint Surg Am 2012;94:1839-44.

35. Si HB, Zeng Y, Shen B, et al. The influence of body mass index on the outcomes of primary total knee arthroplasty. Knee Surg Sports Traumatol Arthrosc 2015;23:1824-32.

36. Jämsen $E$, Nevalainen $P$, Eskelinen $A$,et al. Obesity, diabetes, and preoperative hyperglycemia as predictors of periprosthetic joint infection: a single-center analysis of 7181 primary hip and knee replacements for osteoarthritis. J Bone Joint Surg Am 2012;94:e101.

37. Groen VA, van de Graaf VA, Scholtes VA,et al. Effects of bariatric surgery for knee complaints in (morbidly) obese adult patients: a systematic review. Obes Rev 2015;16:161-70.

38. Lui M, Jones CA, Westby MD. Effect of non-surgical, non-pharmacological weight loss interventions in patients who are obese prior to hip and knee arthroplasty surgery: a rapid review. Syst Rev 2015;4:121.

39. Kahn TL, Snir N, Schwarzkopf R. Does Body Mass Index Decrease Over Time Among Patients Who Undergo Total Knee Arthroplasty Compared to Patients With Osteoarthritis? Data From the Osteoarthritis Initiative. J Arthroplasty 2015. doi: 10.1016/j.arth.2015.11.031.

40. Duthon VB, Ozturk M, El-Achachi S, et al. Effets délétères du tabagisme sur l'appareil musculo-squelettique. Rev Med Suisse 2014;10:1466-71.

41. Singh JA, Schleck C, Harmsen WS, et al. Current tobacco use is associated with higher rates of implant revision and deep infection after total hip or knee arthroplasty: a prospective cohort study. BMC Med 2015;13:283. 
42. Duchman KR, Gao Y, Pugely AJ, et al. The Effect of Smoking on Short-Term Complications Following Total Hip and Knee Arthroplasty. J Bone Joint Surg Am 2015;97:1049-58.

43. Kapadia BH, Johnson AJ, Naziri Q, et al. Increased revision rates after total knee arthroplasty in patients who smoke. J Arthroplasty 2012;27:16901695.e1.

44. Nwachukwu BU, Gurary EB, Lerner V, et al. Effect of smoking and soft tissue release on risk of revision after total knee arthroplasty: a case-control study. BMC Musculoskelet Disord 2015;16:245.

45. Singh JA, Houston TK, Ponce BA,et al. Smoking as a risk factor for short-term outcomes following primary total hip and total knee replacement in veterans. Arthritis Care Res 2011;63:1365-74.

46. Møller AM, Villebro N, Pedersen T, et al. Effect of preoperative smoking intervention on postoperative complications: a randomised clinical trial. Lancet 2002;359:114-7.

47. Smith TO, Penny F, Fleetcroft R. Medical morbidities in people following hip and knee arthroplasty: data from the Osteoarthritis Initiative. Eur J Orthop Surg Traumatol 2016;26:99-106.

48.Bjorgul K, Novicoff WM, Saleh KJ. Evaluating comorbidities in total hip and knee arthroplasty: available instruments. J Orthop Traumatol 2010;11:203-9.

49. Legré-Boyer V, Boyer T. Examen clinique d'un genou douloureux. Rev Rhum Monogr 2016. doi

50. Collins NJ, Misra D, Felson DT, et al. Measures of knee function: International Knee Documentation Committee (IKDC) Subjective Knee Evaluation Form, Knee Injury and Osteoarthritis Outcome Score (KOOS), Knee Injury and Osteoarthritis Outcome Score Physical Function Short Form (KOOS-PS), Knee Outcome Survey Activities of Daily Living Scale (KOS-ADL), Lysholm Knee Scoring Scale, Oxford Knee Score (OKS), Western Ontario and McMaster Universities Osteoarthritis Index (WOMAC), Activity Rating Scale (ARS), and Tegner Activity Score (TAS). Arthritis Care Res 2011;63(Suppl 11):S208-28.

51. Jenny JY, Diesinger Y. Validation du questionnaire d'Oxford en langue française sur le genou. Rev Chir Orthop Traumatol 2011;97:260-5. 
52. Morvan G, Vuillemin V, Guerini H, et al. L'homme debout. Imagerie. Le système EOS. E-Mem Acad. Chir 2013;12: 006-017.

53. Dowsey MM, Nikpour M, Dieppe $P$, et al. Associations between pre-operative radiographic changes and outcomes after total knee joint replacement for osteoarthritis. Osteoarthritis Cartilage 2012;20:1095-102.

54. Tokarski AT, Patel RG, Parvizi J, Deirmengian GK. Dental clearance prior to elective arthroplasty may not be needed for everyone. J Arthroplasty 2014;29:1729-32.

55.Le Moal G. Faut-il continuer à rechercher une bactériurie asymptomatiquechez les candidats à la pose de prothèse articulaire ? Méd Mal Infect $2015 ; 45: 103-4$.

56. Kunutsor SK, Whitehouse MR, Blom AW, et al. Patient-Related Risk Factors for Periprosthetic Joint Infection after Total Joint Arthroplasty: A Systematic Review and Meta-Analysis. PLoS One 2016;11:e0150866.

57. Debreuve-Theresette A, Diallo S, Siboni R, et al. Infections in Total Hip and Total Knee Arthroplasty: Development of a Score To Assess Endogenous Risk of Surgical Site Infections. Surg Infect 2015;16:794-8.

58. Belkaious L, Neyret $P$, Lustig $S$, et al. Les prothèses unicompartimentales du genou : le pour et le contre. Rev Rhum Monogr 2016. doi

59. Haute Autorité de Santé. Elements concourant à la décision d'arthroplastie du genou et du choix de la prothèse. Février 2013.

60. Le Goux P. Quelle pratique sportive après arthroplastie du genou ? Rev Rhum Monogr 2016. doi

61. Delaunay C. L'information du patient. Rev Chirurgie Orthop Réparatrice 2004;90:134-137.

62. Barlow T, Griffin D, Barlow D, Realpe A. Patients' decision making in total knee arthroplasty: a systematic review of qualitative research. Bone Joint Res 2015;4:163-9.

63. Jayadev C, Khan T, Coulter A, et al. Patient decision aids in knee replacement surgery. Knee 2012;19:746-50. 
64. Jordan RW, Smith NA, Chahal GS, et al. Enhanced education and physiotherapy before knee replacement; is it worth it? A systematic review. Physiotherapy 2014;100:305-12.

65. Wang L, Lee M, Zhang Z, et al. Does preoperative rehabilitation for patients planning to undergo joint replacement surgery improve outcomes? A systematic review and meta-analysis of randomised controlled trials. BMJ Open 2016;6:e009857. 
Tableau 1

Bilan préopératoire avant prothèse de genou : aide à la décision

Interrogatoire

Recherche de facteurs de risque généraux

- Etat cardiorespiratoire

- Etat vasculaire périphérique

- Diabète

- Obésité

- Tabac

- Age

Recherche de comorbidités

ostéoporose

dépression légère ou modérée

cancer

antécédent d'accident vasculaire cérébral

asthme

Examen physique

Evaluation fonctionnelle (scores)

Bilan radiologique

Recherche de foyers infectieux

Facteurs de risqué liés à l'anesthésie

Facteurs de risque liés à la chirurgie

Information du patient 